\title{
DVPW
}

\section{DVPW-Dissertationspreis: 2014 keine Preisvergabe}

\author{
Annette Elisabeth Töller / Klaus-Dieter Wolf / Ruth Zimmerling
}

Eingereicht wurden in diesem Jahr zehn Arbeiten. Davon kamen zwei nicht in Frage, da sie nach Auffassung der Jury thematisch nicht ins Kerngebiet der Politikwissenschaft gehören, eine weitere erfüllte das Kriterium der Veröffentlichung in 2013 nicht. Es wurden also sieben Arbeiten begutachtet, die eine große Bandbreite politikwissenschaftlicher Subdisziplinen und Themen abdeckten. Die von uns ausgewählten Gutachter_innen arbeiteten nicht nur schnell, sie formulierten die Gutachten sowohl mit großer Wertschätzung für die geleistete Arbeit als auch sehr differenziert - den Gutachter_innen an dieser Stelle ganz herzlichen Dank!

Zwar leisten alle Arbeiten einen wichtigen Beitrag zu ihrem jeweiligen Gebiet, und einige erhielten auch exzellente Beurteilungen hinsichtlich der Qualität der empirischen Evidenz oder der Datenanalyse. Allerdings wurde keine der eingereichten Arbeiten als für ein breiteres Spektrum politikwissenschaftlicher Forschung so relevant und zugleich originell im theoretischen Ansatz und Argument beurteilt, dass sie einen wesentlichen Fortschritt für das Fach oder wesentliche Teile des Fachs erbringt. Die Entscheidung, daher in diesem Jahr keinen Preis zu vergeben, wurde eindeutig von den externen Gutachten gestützt (keines votierte uneingeschränkt dafür, die jeweils begutachtete Arbeit für den Preis vorzuschlagen) und von der Jury in großer Übereinstimmung getroffen. 